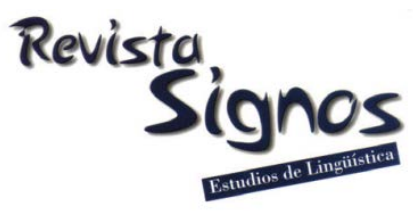

\title{
Textos de identidad digitales: Una valiosa herramienta para el estudio de la construcción de la identidad y el aprendizaje lingüístico en ILE 1
}

\section{Digital texts of identity: A valuable tool for the study of identity construction and language learning in EFL}

\author{
María Dolores García Pastor \\ UNIVERSITAT DE VALÈNCIA \\ ESPAÑA \\ maria.d.garcia@uv.es
}

Recibido: 08-IX-2016 / Aceptado: 15-V-2017

\section{Resumen}

El presente trabajo se centra en el análisis de la identidad en textos de identidad digitales producidos por estudiantes de inglés como lengua extranjera (ILE). Este estudio tiene como objetivo general promover el uso de estos textos como estrategia del enfoque de enseñanza para la transferencia lingüística y el éxito académico (Cummins, 2001, 2005b, 2006) y el refuerzo de la relación familia-escuela (Coll \& Falsafi, 2010). Los textos identitarios permiten a los estudiantes crear y evaluar sus identidades, de modo que el alumno toma conciencia de quién es como aprendiz de lenguas, afianzando las distintas posiciones que conforman su identidad, estableciendo relaciones con identidades de competencia que desea, y rechazando posiciones negativas que le silencian a la hora de comunicarse en la lengua meta. Ello promueve el desarrollo de sus habilidades cognitivas de competencia lingüística académica (CALPS), y en el caso de textos de identidad digitales, de su competencia digital. Para nuestro estudio, se recogieron un total de 51 textos de identidad digitales, los cuales han sido analizados desde una perspectiva 'posicionante' (Davies \& Harré, 1990), combinada con un análisis temático y dialógico/performativo (Block, 2010), y un enfoque cuantitativo descriptivo. Los resultados muestran un equilibrio en la presencia de los posicionamientos reflexivo e interactivo en los textos, con el primero emergiendo a través de distintas posiciones de sujeto en ocasiones opuestas, que se constituían en actos descriptivos y justificativos, y el segundo adoptando la forma de interacciones directas e indirectas con el espectador.

Palabras Clave: Identidad, textos de identidad, perspectiva posicionante, enseñanza y aprendizaje lingüístico, ILE. 


\begin{abstract}
The present study focuses on the analysis of identity in digital texts of identity produced by English as a foreign language (EFL) students. The general aim of this study is to promote the use of these texts as a strategy of the teaching for linguistic transfer and academic expertise approach (Cummins, 2001, 2005b, 2006) and the reinforcement of the family-school relationship (Coll \& Falsafi, 2010). Identity texts enable students to build and evaluate their identities, so that they become aware of who they are as language learners, consolidating the different positions that shape their identities, establishing relationships with identities of competence they desire, and rejecting negative positions that silence them when communicating in the target language. This promotes the development of their cognitive and academic language proficiency skills (CALPS) and, in the case of digital texts of identity, their digital competence. For this study, a total of 51 digital texts of identity were collected and analyzed following a 'positioning' perspective (Davies \& Harré, 1990) that was combined with thematic and dialogic/performative analysis (Block, 2010), and a descriptive quantitative approach. Results illustrate a balance in the presence of reflexive and interactive positioning in the texts, with the former emerging through different subject positions that were occasionally opposed, and were enacted through descriptive and justifying acts, and the latter adopting the form of direct and indirect interactions with the viewer.
\end{abstract}

Key Words: Identity, identity texts, positioning perspective, language teaching and learning, EFL.

\title{
INTRODUCCIÓN
}

Los textos de identidad conforman un tipo de discurso que aborda tanto la afirmación de la identidad como el desarrollo de la lectoescritura. La investigación en alumnado bilingüe y multilingüe, aprendiz de inglés como lengua extranjera (ILE), ha probado que ambos aspectos son igualmente importantes para el éxito académico (Cummins, Bismilla, Chow, Cohen, Giampapa, Leoni, Sandhu \& Sastri, 2005; Bernhard, Cummins, Campoy, Ada, Winsler \& Bleiker, 2006; Giampapa, 2010). En concreto, los textos de identidad digitales han demostrado ser altamente eficaces para el desarrollo de las habilidades académicas multilingües y multimodales de los alumnos.

A pesar de ello, el concepto de identidad y su vinculación al éxito académico ha sido generalmente ignorado en las prácticas pedagógicas y en la planificación curricular (Coll \& Falsafi, 2010; Cummins et al., 2005; Cummins \& Early, 2011; Cummins, Hu, Markus \& Montero, 2015; Esteban-Guitart \& Moll, 2014; Zacarias, 2012). Se añade el hecho de que la mayoría de investigaciones sobre textos de identidad y conceptos relacionados, como por ejemplo, la noción de 'fondos de conocimiento', a saber, cuerpos de conocimientos y destrezas culturalmente desarrollados, históricamente acumulados, y socialmente distribuidos, esenciales para el funcionamiento y bienestar familiar e individual (González, Moll \& Amanti, 2005), y 'fondos de identidad', es decir, los fondos de conocimiento que internalizamos y utilizamos para definir y 
comprender nuestra identidad (Esteban-Guitart, Oller \& Vila, 2012; Esteban-Guitart \& Saubix, 2013; Esteban-Guitart \& Moll, 2014), se han llevado a cabo con escolares procedentes de grupos marginales (sobre todo inmigrantes) en detrimento de otras poblaciones estudiantiles como la que participa en este estudio: estudiantes universitarios de lengua extranjera (LE) de clase media y trabajadora.

Además, los estudios de identidad en este tipo de alumnado aprendiz de ILE se han centrado en autobiografías lingüísticas y diarios de clase (Zacarías, 2012; Yang, 2013), los cuales no maximizan la conexión entre la vida de los estudiantes y su contexto educativo tanto como los textos identitarios, y en consecuencia, no potencian del mismo modo la contextualización, el aprendizaje significativo y la relación entre las experiencias de los alumnos dentro y fuera de dicho contexto. El presente trabajo pretende pues atender a estas limitaciones enfatizando la utilidad de estos textos para alcanzar el éxito académico en este tipo de alumnos, así como la importancia del constructo 'identidad' en el aprendizaje lingüístico (Norton \& Toohey, 2011; Norton, 2010, 2013). Por tanto, abogamos por una pedagogía que i) favorezca la comprensión y el desarrollo de la identidad del aprendiz, su progreso lingüístico académico y la transferencia interlingüística, y ii) fortalezca la relación familia-escuela (Coll \& Falsafi, 2010; Cummins, 2001, 2005a, 2006).

Para ello, en este trabajo exploramos la identidad que presentan estudiantes universitarios de ILE en textos de identidad digitales (TIDs), partiendo de la noción de identidad desarrollada por Norton y colaboradores en adquisición de segundas lenguas (ASL) (Norton \& Toohey, 2011; Norton, 1995, 2000, 2010, 2013; Darvin \& Norton, 2015), y de la teoría 'posicionante' de Davies y Harré (1990). Nuestro análisis se centra en las relaciones que nuestros estudiantes establecen entre su identidad y las llamadas 'identidades de competencia' (identities of competence), las cuales se asocian a la lectoescritura y al desarrollo académico global (ej., la traducción simultánea) (Manyak, 2004), y entre esta e identidades que rechazan, las cuales se relacionan con posiciones silenciadoras que atentan contra su libertad de expresión. Así pues, en este estudio pretendemos averiguar qué posiciones describen la identidad de nuestros estudiantes en sus TIDs; cómo se relacionan con identidades de competencia e identidades silenciadoras; y cuáles son las prácticas discursivas y recursos que las sustentan.

\section{Marco teórico}

\subsection{Textos de identidad: El concepto de identidad}

Los textos de identidad son el producto del trabajo creativo realizado por estudiantes en el contexto del aula y el espacio pedagógico orquestado por el profesor para tal fin (Cummins \& Early, 2011). Dichos textos pueden adoptar distintas formas (escritas, orales, musicales, visuales, teatrales, etc.) combinables en un todo multimodal. En ellos, los alumnos envisten sus identidades, afirmando así su autoimagen, lo cual contribuye a su progreso en el aprendizaje y dominio de la lengua: 
"The identity text then holds a mirror up to students in which their identities are reflected back in a positive light. When students share identity texts with multiple audiences (peers, teachers, [...] etc.) they are likely to receive positive feedback and affirmation of self in interaction with these audiences" (Cummins \& Early, 2011: 3).

Los textos de identidad pueden ser bilingües o multilingües, y en general, incorporan la escritura creativa y el relato digital, que actúan como un vehículo mediante el cual los alumnos pueden rechazar estereotipos negativos de sí mismos o de otros, y construir al mismo tiempo una identidad de sujeto competente y eficaz que fomente su desarrollo académico (García-Pastor, en prensa). Por tanto, no solo sirven para que el autor presente una imagen de sí mismo a sí mismo y al espectador, sino también para que asocie dicha imagen a identidades de competencia que desea alcanzar, y rechace otras que no desea (Norton, 1995, 2000; Cummins, 2006; Darvin \& Norton, 2015).

En el contexto de la enseñanza y el aprendizaje de lenguas, definimos el concepto de identidad como la relación que el aprendiz entiende que tiene, mantiene e imagina con el mundo, la construcción de la misma a través del tiempo y del espacio, y la proyección de esta en el futuro (Norton, 1995, 2000, 2013). La relación entre identidad y aprendizaje lingüístico ha sido investigada desde distintos enfoques, principalmente desde perspectivas socio-culturales y post-estructuralistas de índole socio-constructivista (Block, 2010; Norton, 2010; Norton \& Toohey, 2011). Desde estas perspectivas, la identidad constituye un constructo fluido, multidimensional, a veces contradictorio, que se negocia y se construye socialmente en y a través del discurso, que depende del contexto, y que implica una lucha discursiva del aprendiz para alcanzar la identidad o identidades que desea en un contexto caracterizado por relaciones de desigualdad (Baxter, 2016).

En concreto, en este trabajo hemos seguido la teoría 'posicionante' desarrollada por Davies y Harré (1990), por la cual la identidad se define en términos de posiciones de sujeto (subject positions), entendidas como la producción discursiva de una diversidad de 'yos', y los actos discursivos a través de los cuales dichas posiciones emergen, a saber, actos de posicionamiento (positioning). Estos autores distinguen además dos tipos de posicionamiento principales: el posicionamiento 'interactivo' (interactive positioning), en el que los enunciados del hablante posicionan al interlocutor a través de la toma de turnos, y el posicionamiento 'reflexivo' (reflexive positioning), en el que el mismo hablante actúa para posicionarse. Desde esta teoría, los individuos son posicionados por las prácticas discursivas que llevan a cabo, las cuales proporcionan las posiciones de sujeto desde las que pueden hablar, y los recursos que las sustentan, dando prioridad así a determinadas posiciones sobre otras. Ello no excluye la capacidad activa del individuo para elegir entre las distintas posiciones que se le presentan, o resistirse a aquellas que le son adscritas. 
Así pues, en su construcción de la identidad, los aprendices de ILE indican las distintas posiciones desde las cuales pueden participar en la lengua extranjera, y a su vez muestran el acceso que tienen a los distintos recursos y prácticas que les permiten construir sus identidades en el modo en que lo hacen (Zacharias, 2012; Yang, 2013; García-Pastor, en prensa). El reto para los educadores es pues explorar qué identidades ofrecen al alumnado mayores oportunidades para la participación social en el idioma extranjero, y cuáles le silencian para poder llevar a cabo prácticas pedagógicas que potencien las primeras y disminuyan las segundas (Norton, 2010; Norton \& Toohey, 2011).

\subsection{Textos de identidad: Prácticas pedagógicas}

Los textos de identidad son una estrategia fundamental del modelo de enseñanza para la transferencia lingüística y el desarrollo lingüístico académico propuesto por Cummins (2001, 2005b, 2006). Este modelo se basa en la conocida 'hipótesis de la interdependencia lingüística', que establece que cada lengua contiene características superficiales a las que subyacen competencias que son comunes y, por tanto, transferibles entre lenguas (Cummins 1983, 2005b). Estas competencias implican tareas cognitivamente exigentes como la lectoescritura, el aprendizaje de contenidos, el pensamiento abstracto, y la resolución de problemas, que requieren el dominio de un lenguaje complejo como el lenguaje académico. Dichas competencias o habilidades cognitivas de competencia académica y lingüística (cognitive academic language proficiency skills, CALPS) son específicas del contexto escolar, y reflejan la lengua que los niños adquieren y necesitan utilizar eficazmente para poder progresar con éxito en el mismo (Cummins, 2008).

Los textos identitarios contribuyen al desarrollo de las CALPS y señalan la importancia del concepto de identidad y la negociación de la misma en la comprensión de la naturaleza de la interacción en el aula y los procesos de aprendizaje de los estudiantes (Cummins, 2006). En este sentido, dichos textos han sido utilizados principalmente en Canadá, fundamentalmente en centros escolares públicos tanto de Educación primaria como de Educación secundaria; en materias diversas; y con el objetivo de ayudar a alumnos inmigrantes y estudiantes de minorías étnicas (indígenas) en su aprendizaje del inglés, así como en su integración en el sistema educativo y el país de destino en el caso de los primeros (Cummins et al., 2005; Giampapa, 2010; Cummins et al., 2015). En estos contextos, los textos de identidad son producidos tanto en la lengua materna del estudiante como en inglés; suelen ser creados por varios alumnos con la colaboración de padres y profesores, y son generalmente mostrados en eventos públicos organizados en bibliotecas, museos, etc. Así pues, en el panorama canadiense, dichos textos constituyen una medida de atención a la diversidad en las poblaciones estudiantiles mencionadas con el fin último de acercar el currículum y la escuela a estos alumnos y a sus familias. 
En nuestro trabajo, sin embargo, los textos identitarios han sido empleados en un contexto de aprendizaje de inglés como lengua extranjera (ILE) en un centro de educación superior español con estudiantes de clase media y trabajadora futuros docentes de inglés. Estos textos han sido creados de manera individual por cada estudiante y únicamente en la LE (vid. Apéndice). Dichos textos han supuesto una herramienta para la exploración del 'yo', especialmente del 'yo' que aprende lenguas, de modo que los estudiantes encontraran un espacio en el que representar sus múltiples identidades; desarrollar su competencia oral, y reflexionar sobre su propio aprendizaje lingüístico desde un punto de vista crítico (cf. Cummins et al., 2005; Coll \& Falsafi, 2010; Cummins et al., 2015). Por tanto, nuestro uso de dichos textos es más específico que en el panorama canadiense, aunque nuestro objetivo general es el mismo: contribuir al desarrollo de la identidad del alumno y de las CALPS, así como estrechar la relación entre su vida diaria y su formación universitaria. Los textos de identidad facilitan pues el alcance de este tipo de objetivos en mayor medida que las autobiografías lingüísticas y los diarios de clase, generalmente utilizados en estudios sobre identidad en alumnos universitarios de ILE (Zacharias, 2012; Yang, 2013).

\section{Metodología}

\subsection{Corpus y procedimientos para su recogida}

Teniendo en cuenta lo expuesto anteriormente, este trabajo ha sido guiado por las siguientes preguntas de investigación (PIs):

PI ¿Cómo articulan el posicionamiento reflexivo e interactivo la identidad de los estudiantes universitarios de ILE en los TIDs y qué posiciones de sujeto los definen?,

PI ¿Cómo se relacionan dichos posicionamientos y posiciones con identidades de competencia que se asocian al desarrollo de la lectoescritura y al éxito académico, e identidades opuestas de carácter silenciador?

PI ¿Cuáles son las prácticas discursivas y recursos que las sustentan?

Para dar respuesta a estas preguntas, se recogieron un total de 51 textos de identidad digitales producidos por estudiantes universitarios de ILE ( 7 hombres y 44 mujeres) en el contexto de una asignatura concreta del grado de Maestro en Educación primaria de una universidad española. Todos ellos poseían un nivel B2 de inglés en el momento de la investigación, y son hablantes bilingües de españolvalenciano en su mayoría.

Para la producción de dichos textos, se diseñó una consigna por medio de la cual los estudiantes recibieron instrucciones que se referían a aspectos de contenido y de forma. En relación con el contenido, el texto debía cumplir las siguientes condiciones: 
- Centrarse en la descripción bien de una etapa específica del aprendizaje de ILE del estudiante, bien de su experiencia hasta la fecha.

- Incluir una pregunta dramática que guiara la historia y que se respondiera a lo largo de la misma (cf. Robin, 2006; Gregori-Signes, 2014).

- Describir a su autor/a a la luz de los factores que afectan a la adquisición de la lengua extranjera (la edad, la inteligencia, la aptitud, la motivación, etc.).

- Describir el/los estilo/s y estrategias de aprendizaje propios.

En cuanto a forma, los textos debían adoptar un formato digital y no debían exceder los 7 minutos de duración. Su evaluación fue realizada por los propios estudiantes de manera anónima, siguiendo una rúbrica que se adaptó a la tarea (Robin, 2006).

El ‘tipo de formato’ (relato digital, video de Powtoon, Scribe, etc.) y la 'interacción directa/indirecta con el espectador' constituyeron los criterios principales empleados para la clasificación de los textos, identificando así tres tipos principales de TIDs en el corpus, a los que se les han asignado los siguientes códigos (Lampert \& Ervin-Tripp, 1993): texto de identidad personal (TIP), texto de identidad impersonal (TIIMP), y texto de identidad híbrido (TIH). A estos se les ha añadido códigos identificativos del formato (POW para Powtoon, etc.), así como las iniciales de cada estudiante, por ejemplo: TIIMP-POW-NP.

\subsection{Análisis}

El análisis de los TIDs sigue la perspectiva de Davies y Harré (1990), y dos tipos de análisis narrativo relacionados con la identidad y su negociación (Block, 2010): el análisis temático, que focaliza en el contenido de lo que se dice y se corresponde con el análisis llevado a cabo para el posicionamiento reflexivo; y el análisis dialógico/performativo, que pone el énfasis en a quien va dirigido el enunciado del hablante (en nuestro caso, el espectador) y el propósito del mismo, que se corresponde con el análisis realizado para el posicionamiento interactivo. Además de este análisis cualitativo, hemos incluido datos cuantitativos para lograr una interpretación más precisa de los resultados.

El análisis cualitativo se realizó en una fase general en la que se pretendía obtener una visión global del contenido y estructura de los TIDs, y una fase más específica focalizada en i) la detección de temas relevantes como configuradores de categorías identitarias, y ii) tipos de actos comunicativos y recursos lingüísticos por los cuales el autor/narrador se posiciona en su texto reflexivamente y posiciona al espectador interactivamente. Se tuvieron en cuenta macro identidades ya establecidas como la de 'hablante nativo' (Darvin \& Norton 2015), 'hablante intercultural' (House, 2008), 'estudiante' (Fong, Lin \& Engle, 2016), y ‘aprendiz' (Coll \& Falsafi, 2010; Fong et al., 
2016) (Tabla 1). Finalmente, parte de los datos fueron analizados por otro investigador para asegurar la validez de las categorías.

Tabla 1. Macro categorías de análisis.

\begin{tabular}{|c|c|c|}
\hline Categoría & Definición & Ejemplo \\
\hline $\begin{array}{l}\text { Hablante } \\
\text { nativo }\end{array}$ & $\begin{array}{l}\text { Identidad por la cual se atribuye a un } \\
\text { individuo el dominio completo de su L1 en } \\
\text { las destrezas de comunicación oral, hablar, } \\
\text { escuchar, leer y escribir, constituyendo así } \\
\text { el estándar y el ideal de competencia } \\
\text { lingüística a seguir y alcanzar por aquellos } \\
\text { que aprenden su lengua como L2, L3...o } \\
\text { LE, a los cuales no se les atribuye } \\
\text { pertenencia a su comunidad de hablantes. }\end{array}$ & $\begin{array}{l}\text { "I have grown up hearing German during } \\
\text { a big and important part of the day, five } \\
\text { days a week; and since all of the teachers } \\
\text { were native speakers, there was no other } \\
\text { option for us children to try and speak } \\
\text { German if we wanted to communicate." } \\
\text { (TIP-HV) }\end{array}$ \\
\hline $\begin{array}{l}\text { Hablante } \\
\text { intercultural }\end{array}$ & $\begin{array}{l}\text { Identidad por la cual se atribuye a un } \\
\text { individuo conocimiento en otras lenguas y } \\
\text { culturas distintas a la propia, así como una } \\
\text { actitud alejada del etnocentrismo, y una } \\
\text { capacidad de mediar entre todas ellas en } \\
\text { situaciones de comunicación intercultural, y } \\
\text { de redefinir su identidad. }\end{array}$ & $\begin{array}{l}\text { "...and I managed to go to Norway, where } \\
\text { I spent these five last months. It was an } \\
\text { amazing experience which allowed me not } \\
\text { only to improve my English level, but also } \\
\text { to develop other skills [...] I met a lot of } \\
\text { people from different countries which } \\
\text { allowed me to develop and improve my } \\
\text { social skills. I learnt to cook food from } \\
\text { other cultures. I met very good friends } \\
\text { which I'm still in contact." (TIP-AG) }\end{array}$ \\
\hline Estudiante & $\begin{array}{l}\text { Identidad por la cual se atribuye a un } \\
\text { individuo el estudio de cualquier materia, } \\
\text { manifestándose dicho estudio en y a través } \\
\text { del uso de un determinado registro, el cual } \\
\text { incluye la discusión de determinados temas } \\
\text { y el uso de referentes y un vocabulario } \\
\text { específico en el discurso. La atribución de } \\
\text { dicho estudio suele conllevar el aprendizaje } \\
\text { de la materia, aunque no necesariamente en } \\
\text { todos los casos ni en la misma medida. }\end{array}$ & $\begin{array}{l}\text { "In that sense, it is important to mention } \\
\text { that age was an important internal factor } \\
\text { that helped them to develop good } \\
\text { competence. They were able to produce } \\
\text { output because they had received a lot of } \\
\text { comprehensible input from their parents } \\
\text { and from a lot of people of the town, as } \\
\text { Krashen claims." (TIP-AG) }\end{array}$ \\
\hline Aprendiz & $\begin{array}{l}\text { Identidad por la cual se atribuye a un } \\
\text { individuo i) la adquisición y el desarrollo de } \\
\text { conocimientos, habilidades, destrezas, etc., } \\
\text { en situaciones específicas y/o a lo largo de } \\
\text { su vida, así como la conexión de procesos } \\
\text { cognitivos y afectivos, que actúan de } \\
\text { mediadores en experiencias pasadas, } \\
\text { presentes y futuras de igual naturaleza, y ii) } \\
\text { la pertenencia a determinadas comunidades } \\
\text { de práctica como resultado de todo lo } \\
\text { anterior. }\end{array}$ & $\begin{array}{l}\text { "...I didn't learn English just by talking to } \\
\text { others, as I said before, I practiced } \\
\text { listening with music but I used another } \\
\text { way when I discovered the wonderful } \\
\text { world of series: Glee, Teen wolf, Game of } \\
\text { thrones, New girl, Friends, and many } \\
\text { many, many more, are series that I used to } \\
\text { practice listening by watching them in } \\
\text { original version. At first with Spanish } \\
\text { subtitles but then with English subtitles." } \\
\text { (TIP-EV) }\end{array}$ \\
\hline
\end{tabular}




\section{Resultados y discusión}

\subsection{Posicionamiento reflexivo e interactivo $y$ posiciones de sujeto en los TIDs: Constitución y distribución}

En cuanto a nuestra primera pregunta de investigación, en general, el posicionamiento reflexivo aparece combinado con un posicionamiento interactivo, y las posiciones que lo definen incluyen posiciones de aprendiz según el contexto de aprendizaje, características generales de este, y características en relación con el proceso de aprendizaje. Entre las posiciones de aprendiz según el contexto de aprendizaje, se encuentran aquellas que los alumnos identifican con un contexto de aprendizaje formal (aprendiz de inglés en Educación primaria y secundaria, aprendiz de inglés en actividades extraescolares, aprendiz de inglés en clases particulares, y aprendiz de inglés en academia de idiomas), y las que equiparan a un contexto natural (aprendiz de inglés en contexto de inmersión lingüística, bilingüe simultaneo/familiar, hablante intercultural) (Coll \& Falsafi, 2010).

Las posiciones que describen tipos de aprendiz según características generales incluyen las de 'aprendiz activo', 'aprendiz motivado', 'aprendiz pasivo', y 'aprendiz desmotivado'. Aquellas que aluden a tipos de aprendiz según el proceso de aprendizaje contienen las categorías de 'aprendiz visual', ‘aprendiz auditivo', y 'aprendiz dependiente/independiente del contexto', entre las más comunes (Ellis, 2004). Las primeras hacen referencia a identidades relacionadas con situaciones de aprendizaje específicas que los alumnos han experimentado en determinados momentos de su vida, y, por tanto, se englobarían dentro la ‘identidad de aprendiz de proceso’ (learner identity process, LIP) de Coll y Falsafi (2010), mientras que las segundas se refieren a la 'identidad de aprendiz' (learner identity, LI) que se genera a través del tiempo y del espacio (ibid.). De todas estas posiciones que aparecen prácticamente en la totalidad de los textos, los alumnos solo perciben algunas como identidades silenciadoras, identidades de tránsito, e identidades de competencia reales o imaginarias (Manyak, 2004; Norton, 2010; Norton \& Toohey, 2011) (Tabla 2).

Tabla 2. Posicionamiento reflexivo en los TIDs.

\begin{tabular}{|l|l|l|}
\hline \multicolumn{1}{|c|}{ Identidades silenciadoras } & \multicolumn{1}{|c|}{ Identidades de tránsito } & \multicolumn{1}{|c|}{ Identidades de competencia } \\
\hline Aprendiz de inglés en & Aprendiz de inglés en Educación & Aprendiz de inglés en contexto de \\
Educación secundaria: & primaria & inmersión lingüística \\
Aprendiz pasivo & Aprendiz de inglés en actividades & Aprendiz de inglés con \\
Aprendiz desmotivado & extraescolares & competencia oral casi nativa \\
& Aprendiz de inglés en clases & Hablante nativo \\
& particulares & Bilingüe simultáneo/familiar \\
& Aprendiz de inglés en academia & Hablante intercultural \\
& de idiomas & Maestro/a de inglés en Educación \\
& Consumidor/a de material & primaria \\
& audiovisual en lengua inglesa & Aprendiz activo \\
& & Aprendiz motivado \\
\hline
\end{tabular}


Así pues, las identidades silenciadoras incluyen para los estudiantes principalmente la posición de 'aprendiz de inglés en Educación secundaria'. Los alumnos asocian dicha posición con posiciones que describen tipos de aprendiz poco eficaces tales como ‘aprendiz pasivo' y 'aprendiz desmotivado' (cf. Norton, 1995, 2010; Norton \& Toohey, 2011). Por otra parte, identifican mayoritariamente posiciones como 'aprendiz de inglés en Educación primaria', 'aprendiz de inglés en actividades extraescolares', 'aprendiz de inglés en clases particulares', y 'aprendiz de inglés en academia de idiomas' con identidades de tránsito o posiciones que facilitan el alcance de identidades de competencia reales o imaginadas, a las que se añade la posición de 'consumidor/a de material audiovisual en lengua inglesa'. A diferencia de las anteriores, no perciben esta última como una identidad de aprendiz específica (LIP) sino como parte de su identidad como aprendices de inglés (LI) (Coll \& Falsafi, 2010). Finalmente, equiparan identidades de competencia a las posiciones de 'aprendiz de inglés en contexto de inmersión lingüística', 'aprendiz de inglés con competencia oral casi nativa', 'hablante nativo', 'bilingüe simultáneo/familiar', 'hablante intercultural', 'maestro/a de inglés en Educación primaria', 'aprendiz activo', y 'aprendiz motivado' (Norton, 2010; Zacharias, 2012; Cummins et al., 2015). Solo estas dos posiciones junto con 'hablante intercultural' son descritas como identidades de aprendiz específicas (LIP) (Coll \& Falsafi, 2010). El resto emergen como identidades de aprendiz (LI) imaginadas que los alumnos desean alcanzar.

Por su parte, las posiciones que definen el posicionamiento interactivo en los TIDs constituyen distintas categorías de espectadores, entre las que se encuentran: i) un ente genérico a quien se dirige el narrador, equivalente a la 'audiencia que escucha' (overhearing audience) (Bell, 1984), y ii) el profesor y/o los compañeros de clase que van a visionar y evaluar el texto. A estos, el narrador se dirige en ocasiones directamente (ej., “Hello class"), constituyendo así los 'destinatarios' (addressees) del mensaje, o de forma indirecta, de modo que son 'oyentes' (auditors) del mismo (ibid.).

La combinación del posicionamiento reflexivo e interactivo se da de manera equilibrada en 38 textos (74\%), en los que el posicionamiento interactivo se observa en una interacción indirecta que el narrador mantiene con el espectador. El resto de textos enfatizan este posicionamiento mediante conversaciones directas que el primero establece con este último en algún punto del relato $(n=7,14 \%)$ o en la totalidad del mismo $(n=6,12 \%)$. En dichas conversaciones, el espectador bien constituye el único interlocutor, u otro más junto con amigos y familiares del narrador (Figura 1). 


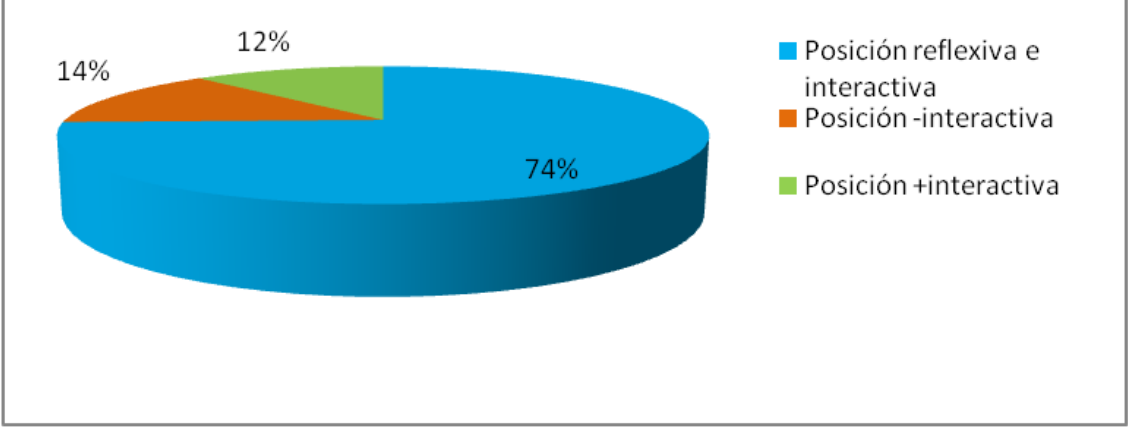

Figura 1. Distribución del posicionamiento reflexivo e interactivo en los TIDs.

Entre los 38 textos que combinan ambas posiciones equilibradamente encontramos diferencias en la manifestación del posicionamiento interactivo según el grado en el que el narrador se dirige más o menos directa o indirectamente al espectador, lo cual evidencia un carácter más personal o impersonal del relato (cf. Robin, 2006; Gregori-Signes, 2014). Así pues, 5 textos (13\%) constituyen relatos creados con imágenes y videos personales, en los que se escucha la voz del alumno contando su historia al espectador. Estos corresponden a la categoría '+voz, +personal'. Otros textos $(\mathrm{n}=15,40 \%)$ cuentan con la voz del narrador, pero contienen pocas imágenes o videos personales. Estos constituyen la categoría ' + voz, -personal'. Once textos (29\%) incluyen la voz del narrador, pero sin imágenes o videos personales (+voz, impersonal). Muchos se basan en el programa Powtoon o presentan un formato de pizarra o espacio similar en donde el narrador escribe, dibuja o coloca imágenes que ilustran su narración; o de fondo de pantalla en el que aparece su historia por escrito. De los textos en los que no se escucha la voz del narrador, 2 $(5,2 \%)$ muestran numerosas imágenes y videos personales de los autores (-voz, +personal), $2(5,2 \%)$ contienen pocas imágenes personales (-voz, -personal), y 2 $(5,2 \%)$ no incluyen ninguna (-voz, impersonal). Finalmente, 1 texto $(2,6 \%)$ incluye la voz del narrador solo en determinados momentos del relato junto con pocas imágenes personales (+/-voz, -personal) (Figura 2). 


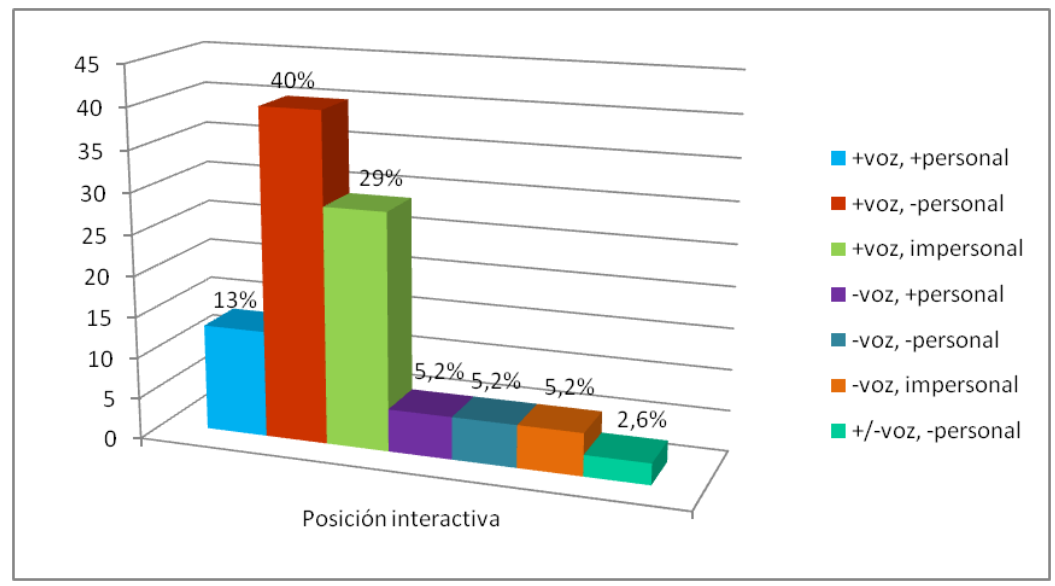

Figura 2. Presencia del posicionamiento interactivo en los TIDs de combinación equilibrada.

\subsection{Posicionamiento reflexivo e interactivo, posiciones de sujeto, e identidades de competencia, de tránsito y silenciadoras: Relaciones y características}

En relación a nuestra segunda pregunta de investigación, los participantes de este estudio muestran su identidad como aprendices de inglés en sus textos principalmente a través de un posicionamiento reflexivo, sustentado en múltiples posiciones de sujeto que ofrecen una imagen fragmentaria de dicha identidad, y en ocasiones, contradictoria (Darvin, 2016). Ello corrobora el carácter no unitario y fluido de este constructo, y el hecho de que los factores del aprendiz que tradicionalmente han sido caracterizados como binarios y mutuamente excluyentes en ASL (ej. aprendiz motivado/no motivado) (Ellis, 2004), revisten mayor complejidad, y, a pesar de ser opuestos, conviven en un mismo individuo (Norton, 1995, 2010; Norton \& Toohey, 2011). El siguiente ejemplo ilustra estos resultados.

(1) TIP-NA

In addition, I went to private lessons for seven years. There, I learnt a lot of English grammar. (Imagen de libros de gramática desde el nivel elemental al nivel intermedio). In those lessons, I was unmotivated, because I was doing fill in the gaps exercises all the time. (Imagen de ejercicios de gramática de rellenar huecos y de elección múltiple).

En este ejemplo la posición de 'aprendiz de inglés en clases particulares' promueve sentimientos positivos en la alumna, ya que contribuye a mostrar una imagen de autoeficacia en su aprendizaje del inglés (I learnt a lot of English grammar) (Cummins et al., 2005; Cummins \& Early, 2011; Cummins et al., 2015), pero a su vez, suscita sentimientos negativos y de rechazo, porque muestra una imagen negativa basada en la 
desmotivación que sintió en su aprendizaje (I was unmotivated because I was doing fill in the gaps exercises all the time). La posición que ejemplifica el extracto constituye una identidad de tránsito.

Las identidades de competencia identificadas por los estudiantes se asocian a una visión específica del éxito académico y el desarrollo de la lectoescritura, basada principalmente en el uso de la lengua inglesa en la comunicación oral en general, y en intercambios interculturales en particular (Zacharias, 2012). Ello coincide con los hallazgos de Manyak (2004), en cuyo trabajo un grupo de escolares hispanos aprendices de inglés y su maestra entendían la capacidad de traducción simultánea como una habilidad de competencia lingüística ventajosa, distintiva y especial, y, en consecuencia, como una identidad de competencia. El ejemplo (2) muestra estos resultados.

(2) TIH-JH

The truth is after the days went by and seeing myself forced to speaking in the academy, my fear of speaking in public started to disappear at the same time as my level of English kept increasing. My self-esteem grew and my attitude started to change.

En este extracto el alumno describe como por medio del esfuerzo logra superar su timidez y comunicarse de manera oral en la lengua extranjera, presentándose a sí mismo como un aprendiz eficaz. De este modo, se identifica con una identidad de competencia que le motiva a seguir esforzándose en su aprendizaje. Este tipo de ejemplos son frecuentes en nuestro corpus, poniendo de relieve la función liberadora y transformadora de los textos de identidad (Cummins et al., 2005; Cummins \& Early, 2011; Cummins et al., 2015).

Por otra parte, los alumnos vinculan las identidades silenciadoras y las posiciones que las sustentan a una 'pedagogía de transmisión' (transmission pedagogy) (Cummins, 2006: 57), a saber, una pedagogía que niega al aprendiz de lenguas el acceso a su uso real, y la posibilidad de atribuir sentido a su proceso de aprendizaje. Esta pedagogía se asocia principalmente a la Educación secundaria, la cual emerge como un contexto institucional que reproduce desigualdades lingüísticas y sociales (cf. Bordieu \& Passeron, 1990):

\section{(3) TIIMP-DH}

But everything changed when I was 17 years old. My extrinsic motivation started to increase in all my subjects due to high school final exams. In this scenario, the pressure of getting good marks to enter a good career become strong. In addition, all lessons including the English one, were boring, repetitive and decontextualized [...] It was in this period that my motivation reached a 
minimum as I decided to leave English lessons out of having failed the FCE exam.

Este ejemplo muestra cómo el estudiante asocia la posición de aprendiz en la Educación secundaria a una identidad silenciadora que se desprende de una pedagogía tradicional que le desmotiva, y de un contexto educativo que está estructurado alrededor de exámenes y notas. Dicho contexto le obliga a adoptar esta identidad que desea rechazar, apareciendo como un contexto coercitivo. Información como la que proporciona este ejemplo, entre otros, puede resultar útil para el docente de idiomas a la hora de implementar prácticas instructivas que permitan a sus alumnos combatir identidades silenciadoras de este tipo (Coll \& Falsafi, 2010; Cummins et al., 2015; Norton \& Toohey, 2011).

Finalmente, los alumnos relacionan el posicionamiento interactivo en los TIDs y las posiciones de sujeto que lo describen con identidades de competencia cuando establecen una relación estrecha con el espectador, y pretenden promover una visión de comunidad en la que inscriben a este y a sí mismos para reforzar su identidad como sujetos competentes, por ejemplo, cuando se posicionan y posicionan al espectador como participantes del curso de didáctica de la lengua extranjera en el que han producido sus textos (Norton, 2010). Sin embargo, vinculan dicho posicionamiento y posiciones de sujeto a identidades silenciadoras, cuando se erigen como expertos en y de sus relatos, adscribiendo indirectamente una posición de sujeto inexperto al espectador, especialmente cuando remarcan su condición de autores además de narradores (Cummins et al., 2015).

\subsection{Posicionamiento reflexivo e interactivo $y$ posiciones de sujeto en los TIDs: Prácticas discursivas y recursos}

En cuanto a nuestra tercera pregunta de investigación, el posicionamiento reflexivo y las posiciones que lo definen se materializa en descripciones de estas, descripciones de emociones positivas o negativas hacia las mismas, y justificaciones implícitas y explícitas, así como justificaciones metalingüísticas sobre el contenido o la forma de la historia. La abundancia de estos actos comunicativos no es sorprendente si se tiene en cuenta que este tipo de enunciados ayudan a regular las ambiciones y frustraciones humanas (Antaki, 1994), y permiten al individuo dar coherencia y continuidad a un ‘yo' fragmentado y discontinuo (Coll \& Falsafi, 2010), reproduciendo así la ideología dominante del 'yo' unitario y auto-contenido que caracteriza a nuestra cultura occidental (Sampson, 1993).

En el siguiente ejemplo, la narradora del texto describe su identidad de manera general al espectador, contando su historia sobre cómo ha vivido, e intentando así producir algún tipo de coherencia entre las distintas posiciones de sujeto que la definen (Baxter, 2016). 
(4) TIP-AG

[...] I was born on 11 of April of 1995 in a little town called Benigembla. It is surrounded by beautiful mountains where fresh air can be breathe (unintelligible) it is near the nature and hardly any car is on the streets. When I started school my family and I moved on to another city called Denia.

Las prácticas discursivas y los recursos que subyacen al posicionamiento interactivo y las posiciones de sujeto que lo integran se basan, por un lado, en preguntas del tipo 'How did I became a language learner?' o 'why did I decide to learn English?'. Estas preguntas aparecen de forma escrita y oral, y se emplean para potenciar el interés del espectador por la historia e incrementar la tensión dramática de la misma (Gregori-Signes, 2014; Robin, 2016). Dicho posicionamiento y las posiciones que lo definen se observan en los TIDs de combinación equilibrada ( $n=38,74 \%$ ) en el uso de enunciados como 'Welcome to my story', 'Me as a language learner', etc., al comienzo del texto; y expresiones como 'thanks for watching!' al final. En ocasiones también emergen por medio de una interacción corta con el espectador, la cual inicia el relato, y se caracteriza por aperturas conversacionales en forma de presentación o saludo, acompañadas de enunciados que i) afirman la identidad del autor como aprendiz de inglés o de lenguas, ii) contextualizan la historia, iii) anticipan su contenido, e incluso iv) expresan el deseo de que la narración sea placentera:

(5) TIP-ABC

Hello, as you know me, my name is A and I'm going to show you my life experience as an EFL learner. I hope that you enjoy my video.

En los textos que acentúan el posicionamiento interactivo $(n=7,14 \%)$, este emerge a través de conversaciones directas con el espectador en medio o al final del relato, que aparecen en algunos casos precedidas de una pregunta que el narrador plantea y que pretende resolver en la interacción con este último; mientras que en otros sirven para marcar una parte distinta de la historia en términos temporales, introducir una anécdota u opinar respecto a un tema relacionado con ASL (ibid.). El siguiente ejemplo muestra una conversación entre la narradora del relato y el espectador, que da respuesta a una pregunta que esta plantea sobre si se sentía motivada en las clases de inglés del instituto.

\section{(6) TIH-POW-MH}

(Imagen con la pregunta: Were we motivated in the High school's lessons?). We were not, for example she thought that if we spoke in English, for example, during a dialogue, ooor playing games, it wasn't a way of learning. She thought that we were only playing. Furthermore, she told us we weren't there to play; we were there to learn. Soo, I think this is a way of desmotivating us [...]. 
Finalmente, en los textos en los que el posicionamiento interactivo constituye la totalidad del relato $(\mathrm{n}=6,12 \%)$, el narrador se sitúa frente a la cámara y establece una conversación continua con el espectador por medio de la cual cuenta su historia. Así pues, los posicionamientos reflexivo e interactivo se construyen fundamentalmente por medio de descripciones y actos justificativos de diversa índole. Este último además se materializa a través de preguntas, enunciados que constituyen saludos y despedidas al principio y final de los textos, y conversaciones más o menos extensas y directas o indirectas con el espectador.

Además de estas prácticas discursivas y recursos, se observa el predominio de un estilo discursivo afectivo característico de algunas formas de comunicación digital de base textual, marcado por un alto grado de intensificación que se aprecia en el empleo de signos de puntuación no normativo (Why did I decide to learn English??!!); palabras o letras en mayúscula; abundancia de elementos intensificadores (very, really, etc.); cuantificadores extremos (everyone, all); repeticiones diversas (cf. Jones \& Hafner, 2012; Page, 2012); y un registro coloquial más común del habla que de la escritura resultante de la transformación que ha sufrido el lenguaje a causa de la tecnología (Darvin, 2016).

\section{CONCLUSIONES}

Este estudio explora la identidad que presentan y construyen discursivamente estudiantes universitarios de ILE en textos de identidad digitales producidos en el contexto de una asignatura concreta del Grado de Maestro/a en Educación primaria (inglés) de una universidad española. En relación con los posicionamientos reflexivo e interactivo, las distintas posiciones de sujeto que adoptan los alumnos en la construcción del 'yo', y las identidades de competencia, de tránsito y silenciadoras con las que se asocian (PI1 y PI2), los alumnos presentan en general una identidad no unitaria, que en ocasiones incluye posiciones de sujeto contradictorias, combinando para ello los posicionamientos reflexivo e interactivo mayoritariamente de manera equilibrada. El posicionamiento reflexivo se relaciona con distintas posiciones de sujeto que los estudiantes identifican con: i) identidades silenciadoras, las cuales incluyen generalmente posiciones de aprendiz de inglés en la Educación secundaria; ii) identidades de tránsito, las cuales constituyen el medio para poder alcanzar identidades de competencia y se refieren principalmente a posiciones con características o elementos constitutivos de naturaleza opuesta; y finalmente, iii) identidades de competencia, que aluden fundamentalmente al uso eficiente de la lengua inglesa como medio de comunicación oral e intercultural.

El posicionamiento interactivo correspondiente al plano dialógico/performativo en el estudio del concepto identidad, se sustenta en posiciones que describen distintos tipos de espectadores tales como un ente genérico o el docente y/o los compañeros 
de clase. Estos tipos de espectadores se vinculan en ocasiones a identidades de competencia, y en otras a identidades silenciadoras. El uso de determinadas prácticas discursivas y recursos lingüísticos sostienen este posicionamiento y el posicionamiento reflexivo en los textos analizados (PI3). En el caso del posicionamiento interactivo, estos constituyen intercambios comunicativos del alumno con el espectador, que se producen a través de preguntas de diversa índole, enunciados cortos al principio y al final de los textos a modo de saludos y despedidas, conversaciones más o menos extensas y directas o indirectas, y toda una serie de actos descriptivos y justificativos que también sustentan el posicionamiento reflexivo.

Entre algunas de las limitaciones de este estudio, destacan un apoyo cuantitativo más sólido de los resultados, así como una menor intervención en la consigna para la producción de los TIDs por parte del profesor/investigador. Futuros trabajos sobre construcción de identidad y aprendizaje lingüístico en aprendices de ILE por medio de estos textos podrían abordar estas cuestiones e implementar tratamientos longitudinales en el empleo de los mismos, además de incorporar otros métodos de recogida de datos y análisis cualitativo que permitieran obtener mayor información identitaria sobre los alumnos tales como entrevistas, el 'círculo significativo' y el autorretrato (Esteban-Guitart \& Moll, 2014). La comparación entre el uso de textos de identidad y otras herramientas similares para explorar la construcción de la identidad de los aprendices podría ser objeto de investigación, así como la relación entre el empleo de dichas herramientas y los resultados de aprendizaje.

Consideramos que los hallazgos de este trabajo podrían ser útiles para el docente de idiomas a la hora de planificar y diseñar intervenciones didácticas que ofrezcan al estudiante la posibilidad de conocer y aprender a usar elementos lingüísticos y estructuras discursivas diversas, que le permitan, por un lado, luchar contra identidades silenciadoras con las que se identifica, y por otro, ocupar posiciones más ventajosas para la participación social y cultural en la lengua meta tanto dentro como fuera del aula. Asimismo, este estudio muestra el valor del empleo de textos identitarios en el aula de ILE como herramienta de refuerzo de la identidad del estudiante, ya que le brindan la posibilidad de sentirse escuchado, atribuirse valor personal, y potenciar sentimientos de orgullo hacia sí mismo. El uso de dichos textos puede así contribuir a revertir la percepción negativa que tienen algunos alumnos sobre el contexto educativo y las prácticas pedagógicas que les rodean, ayudándoles a percibir dicho contexto como un espacio más justo y positivo, y experimentar la enseñanza-aprendizaje como más placentera y significativa. 


\section{REFERENCIAS BIBLIOGRÁFICAS}

Antaki, C. (1994). Explaining and arguing: The social organization of accounts. Londres: Sage.

Baxter, J. (2016). Positioning language and identity: Poststructuralist perspectives. En S. Preece (Ed.), The Routledge handbook of language and identity (pp. 34-49). Londres: Routledge.

Bell, A. (1984). Language style as audience design. Language in Society, 13(2), 145-204.

Bernhard, J. K., Cummins, J., Campoy, F. I., Ada, A. F., Winsler, A. \& Bleiker, C. (2006). Identity texts and literacy development among preschool English language learners: Enhancing learning opportunities for children at risk for learning disabilities. Teachers College Record, 108(11), 2380-2405.

Block, D. (2010). Researching language and identity. En B. Paltridge \& A. Phakti (Eds.), Continuum Companion to Research Methods in Applied Linguistics (pp. 337347). Londres: Continuum.

Bordieu, P. \& Passeron, J-C. (1990). Reproduction in Education, Society and Culture. Londres: Sage.

Coll, C. \& Falsafi, L. (2010). Learner identity: An educational and analytical tool. Revista de Educación, 35(3), 211-233.

Cummins, J. (1983). Interdependencia lingüística y desarrollo educativo de los niños bilingües. Infancia y Aprendizaje, 21, 37-61.

Cummins, J. (2001). Negotiating identities: Education for empowerment in a diverse society. Los Ángeles: California Association for Bilingual Education.

Cummins, J. (2005a). Teaching for cross-language transfer in dual language education: Possibilities and pitfalls [en línea]. Disponible en: http:// http://www.tesol.org/docs/default-source/new-resource-

library/symposium-on-dual-language-education-3.pdf?sfvrsn $=0$

Cummins, J. (2005b). La hipótesis de interdependencia 25 años después: La investigación actual y sus implicaciones para la educación bilingüe. En D. Lasagabaster \& J. Sierra (Eds.), Multilingüismo y multiculturalismo en la escuela (pp. 113-132). Barcelona: ICE-HORSORI y Universitat de Barcelona.

Cummins, J. (2006). Identity texts: The imaginative construction of self through multiliteracies pedagogy. En O. García, T. Skutnabb-kambas \& M. Torres Guzman (Eds.), Imagining multilingual schools: Language in education and glocalization (pp. 51-68). Clevedon: Multilingual Matters. 
Cummins, J. (2008). BICS and CALP: Empirical and theoretical status of the distinction. En B. Street \& N. H. Hornberger (Eds.), Encyclopaedia of language and education, 2: Literacy (pp. 71-83). Nueva York: Springer.

Cummins, J. \& Early, M. (2011). Identity texts: The collaborative creation of power in multilingual schools. UK: Trenthan Books.

Cummins, J., Bismilla, V., Chow, P., Cohen, S., Giampapa, F., Leoni, L., Sandhu, P. \& Sastri, P. (2005). ELL students speak for themselves: Identity texts and literacy engagement in multilingual classrooms [en línea]. Disponible en: http://www.curriculum.org/secretariat/files/ELLidentityTexts.pdf

Cummins, J., Hu, S., Markus, P. \& Montero, M. K. (2015). Indentity texts and academic achievement: Connecting the dots in multilingual school settings. TESOL Quarterly, 49(3), 555-581.

Darvin, R. (2016). Language and identity in the digital age. En S. Preece (Ed.), The Routledge handbook of language and identity (pp. 523-539). Londres: Routledge.

Darvin, R. \& Norton, B. (2015). Identity and a model of investment in applied linguistics. Annual Review of Applied Linguistics, 35, 36-56.

Davies, B. \& Harré, R. (1990). Positioning: The discursive production of selves. Journal for the Theory of Social Behaviour, 20(1), 43-63.

Ellis, R. (2004). Individual differences in second language learning. En C. Elder \& A. Davies (Eds.), Handbook of applied linguistics (pp. 525-551). Oxford: Blackwell.

Esteban-Guitart, M. \& Moll, L. (2014). Funds of identity: A new concept based on the funds of knowledge approach. Culture \& Psychology, 20(1), 31-48.

Esteban-Guitart, M. \& Saubix, X. (2013). La práctica educativa desde la perspectiva de los fondos de conocimiento e identidad. Teoría de la Educación, 25(2), 189-211.

Esteban-Guitart, M., Oller, J. \& Vila, I. (2012). Vinculando escuela, familia y comunidad a través de los fondos de conocimiento e identidad: Un estudio de caso con una familia de origen marroquí. Revista de Investigación en Educación, 10(2), 21-34.

Fong, C. J., Lin, S. \& Engle, R. A. (2016). Positioning identity in computer-mediated discourse among ESOL learners. Language Learning \& Technology, 20(3), 142158.

García-Pastor, M. D. (en prensa). Learners' identities at stake: Digital identity texts in the EFL classroom, Language Value, 9. 
Giampapa, F. (2010). Multiliteracies, pedagogy and identities: Teacher and student voices from a Toronto elementary school. Canadian Journal of Education, 33(2), 407-431.

González, N., Moll, L. \& Amanti, K. (2005). Funds of knowledge: Theorizing practices in households, communities, and classrooms. Mahwah, NJ: Lawrence Erlbaum.

Gregori-Signes, C. (2014). Digital storytelling and multimodal literacy in education. Porta Linguarum, 22, 237-250.

House, J. (2008). What is an 'intercultural speaker'?. En E. Alcón Soler \& M. P. Safont Jordá (Eds.), Intercultural language use and language learning (pp. 7-21). Nueva York: Springer.

Jones, R. H. \& Hafner, C. A. (2012). Understanding digital literacies: A practical introduction. Londres: Routledge.

Lampert, M. D. \& Ervin-Trip, S. M. (1993). Structured coding for the study of language and social interaction. En J. A. Edwards \& M. D. Lampert (Eds), Talking data: Transcription and coding in discourse research (pp. 169-206). Hillsdale: Lawrence Erlbaum.

Manyak, P. C. (2004). "What did she say?" Translation in a primary-grade English immersion class. Multicultural Perspectives, 6(1), 1-218.

Norton, B. (1995). Social identity, investment and language learning. TESOL Quarterly, 29(1), 9-31.

Norton, B. (2000). Identity and language learning: Gender, ethnicity and educational change. Harlow: Pearson Education.

Norton, B. (2010). Language and identity. En N. Hornberger \& S. McKay (Eds.), Sociolinguistics and language education (pp. 349-369). Bristol: Multilingual Matters.

Norton, B. (2013). Identity and language learning: Extending the conversation. Bristol: Multilingual Matters.

Norton, B. \& Toohey, K. (2011). Identity, language learning, and social change. Language Teaching, 44(4), 412-446.

Page, R. E. (2012). Stories and social media: Identities and interaction. Londres: Routledge.

Robin, B. R. (2006). The educational uses of digital storytelling [en línea]. Disponible en: https://dsresources.pbworks.com/f/Educaional-Uses-DS.pdf

Sampson, E. E. (1993). Celebrating the other: A dialogic account of human nature. Boulder: Westview Press. 
Yang, S. (2013). Autobiographical writing and identity in EFL education. Londres: Routledge.

Zacarias, N. T. (2012). EFL students' understanding of their multilingual English identities, Electronic Journal of Foreign Language Teaching, 9(2), 233-244.

\section{ANEXO}

Extracto de un TID del corpus. Incluye también música y la voz de la narradora.

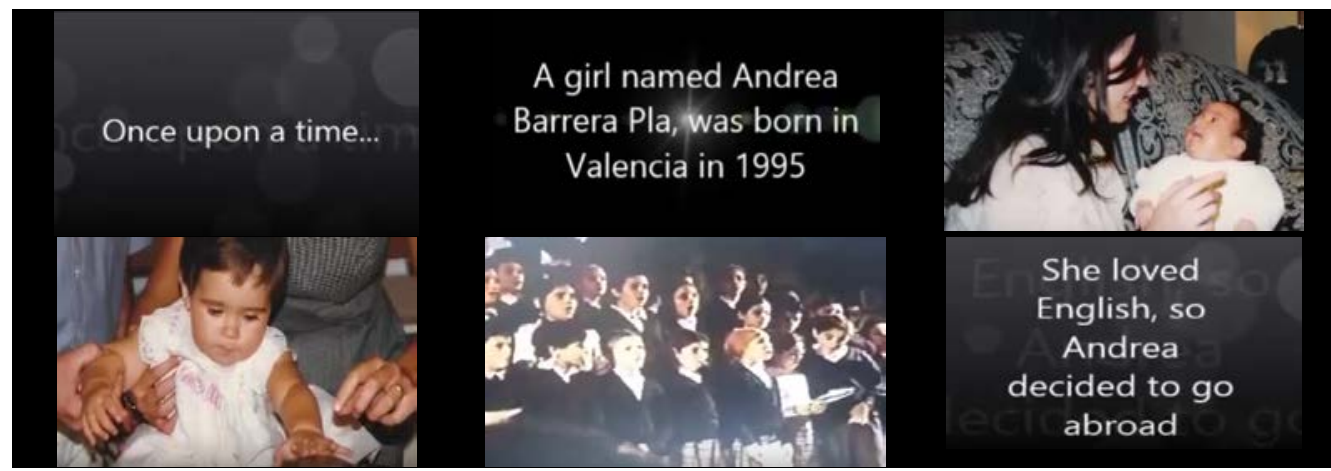

\section{NOTA}

1 Este trabajo se ha desarrollado en el marco de un Proyecto de innovación docente subvencionado desde el curso 2015-16 con título "El uso de textos de identidad en inglés con futuro/as maestros/as de primaria: enseñar para la transferencia interlingüística en educación superior" (ref. UV-SFPIE-RMD15-314975), así como en el seno del grupo de investigación GIEL (Grupo de investigación sobre enseñanza de lenguas). Se agradecen los valiosos comentarios de los dos revisores anónimos de este artículo, así como las sugerencias del profesor Ronan Miller en una versión anterior del mismo. 